78

so; dipendono perciò dalla velocità delle variazioni e dalla quantità d'elettricità messa in moto nello stato rariabile.

Allorchè l' interruzione non è molto breve, le forze elettromotrici indotte successivamente di segno contrario possono svilupparsi separatamente. Le lora intensità s' avvicinano all eguaglianza e quindi s'annullano i loro effetti quanto più le interruzioni sono brevi.

La colrente indotta all'interruzione presenta la sua massima intensità al momento dell' interruzione e quindi decresce in un modo continuo, ciò che fa che la scossa eccitata in quell'istante è più forte. Avviene il contrario colla corrente della pila perchè la variazione dell'intensità è più brusca al chiudere che all' aprire del circuito.

Sarebbe importante di continuare questi studii operando sopra i nervi semplici, sopra nervi misti e ganglionari e sopra diversi animali.

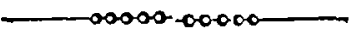

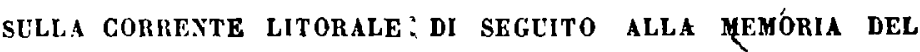
COMMENDator PaLEOCAPA, LETTA ALL' 'STITUTO LOMBaRdo SELLA TORXATA DEL 2/4 MAGglo 1860; MEMORIA DEL PROF, CAV. MIALRIZIO BRIGHENTI.

(Estratta dal Vol. XI. delle Memorie dell' Ac. delle Scienze dell' Istituto di Bologna).

Sono di grandissimo peso le considerazioni che il chiaro paleocapa ha soggiunte sulla corrente litorale nell' istituto Lombardo, facendo onor grande a quanto io ne scrissi due anni sono in questo nostro Bolognese.

Si tratta di determinare, se quella corrente influisca, o no, o quanto sulla direzione delle fosse subacquee, e sull' insabbiasnento delle foci, e però con quali opere artificiali si possa ren- 
derne innocui gli effetti, o mitigarli ad agevolare l'ingresso delle navi ne' porti delle spiagge sottili.

E pershè quell' esimio idraulico va in cerca del vero, e non d'altro; io che dissento da lui, mi studierò d'imitarlo, e non avrò troppa guerra a far tacere l'amore delle mie diverse opinioni, perchè mi sento quanto prossimo all'errore, tanto lontano da presunzione.

Che quella corrente vi sia, oltre il consenso antichissimo degl'ingegneri e degli uomini di mare, non è alcuno che dubiti; ed è generalmente ammesso che percorra tre o quattro miglia venete in 24 ore (M. ${ }^{i}$. 06, o M. 0.07 . ogni $\left.1^{\prime \prime}\right)$, come risulta dalle osservazioni del Montanari (che la credeva anche meno di tre miglia lungo la costa Friulana), e confermate dopo, come si nota nel Portolano dell' Adriatico, pag. 7, 452, 453.

Non so che siano mai state fatte esperienze sulla profondità di questo movimento; legga nello stesso Portolano che la si crede di 6, o 7 metri, deducendola dalla facilita con cui mata andamento, secondo la direzione dei venti che spirano. La qual deduzione procede forse dall'aver osservato che di 6,07 metri debba tenersi la profondità del moto ondoso del mare atto a sconvolgere il fondo.

Sarci inclinato a pensare, che questo moto litorale debba diminuire anzichè crescere dalla superficie al fondo, sembrandomi occasionato dal dislivello superficiale continuo dell'influsso delle acque del Mediterraneo nell' Adriatico. Pare anzi verosimile, che essendo la costa orientale profonda, e di ripe scogliose pochissimo inchinate alla verticale, debbano quelle acque, entrando, di preferenza, e con qualche maggiore vivacità gittarsi da questa parte, tenendosi forse alquanto più alte, che sulla spiaggia bassa occidentale : come accade ne' fumi lungo $i$ froldi, e gli ostacoli verticali, a preferenza delle dolci scarpate, e delle marezzane della parte opposta. Quindi ne deriverebbe che ingolfatesi le acque nel cul-di-sacco fra Trieste e Venczia, per restituirsi al Mediterranco, dovessero scendere lungo il lido occidentale.

Che che sia di ció, questo moto circolare riesce sensibile solamente lungo le coste, attesa la vastissima sezione traversa del Gulfo, come accade dei lievi movimenti circolari impressi 
80

vicino alle pareti, che riescono impercettibili nel mezzo, e salamente sensibili lungo gli orli di un vaso amplissimo.

Sarebbera desiderabili esperienze più dirette, per conoscere perfettamente la natura di questo movimento radente le nostre coste; ma ciò che ne ho riferito, e sappiamo di certo, basta per mio avviso a concludere :

1. ${ }^{\circ}$ Che non può mai essere abile a svolgere il fondo del mare, e tanto meno le punte fluviali ordinariamente più tenaci.

$2 .{ }^{\circ}$ Che muta facilmente andamento, secondo gli ostacoli in cui s'abbatte per via, e allo spirare contrario di venti anche leggieri. Portolano pag. $7,452,453$.

$3 .^{\circ}$ Che pud convogliar seco le tenuissime terbide allorchè il mare è sconvolto, se la direzione del vento è cospirante; se contraria, essendo prevalente l'azione del vento, quelle torbide seguono la direzione contraria.

4. ${ }^{\circ}$ Che quanda il mare è in calma, le sue acque scendono costantemente dalla sinistra alla destra lungo la nostra costa occidentale, e montano similmente lungo la orientale.

Queste conseguenze a me parvero sempre consentanee ai fatti esattamente ( come discorreva nella detta mia Memoria dal 1829 in poi ) e nel 1832 me ne dichiarava prolissamente per servire al chiarissimo Carlini, ehe m'invitava a dire il mio parere sulle opere postume del Tadini (1).

(1) Dalla Biblioteca Italiana di Milano nel fascicolo di Febbrajo 1832 - Sulle opere postume del Tadini .

- La proprielà dell' aequa salata di togliere la tenacilá alle terre sperimentata in piccoli rasi, ed osservata in grande dal Tadini, gli diede occasione di una detta o lunghissima annotazione intorno alla laguna di venezia. Per forza di questa proprietà gli sembrò di poter dare la spiegazione del fatto ch' egli tiene per costantissimo, cioè che nei porti di acqua dolce la fossa attraversante lo scanno contro la bocca segue la direcione del Gume, e in quelli d'acqua salata si rolta alla destra.

- Nel primo caso essendo il foudo oltra la foce coperto dalle acque dolei, e peró tenace a modo da non essere sconvolto dalle onde burrascose del mare, non trova $\mathrm{l}^{\prime}$ Autore cagione che debba alterare la direzione del corso precedente, onde la fossa si cava e rimane diritta, secondo il corso Jel fiume: per contrario ne' porti di acqua salsa, essendo il fondo oltre lo sbocco mobilissimo per la qualità dell' acqua a cui soggiace, le onde mariue lo solicrano, e formasi lo scanno parallelo al lido, e più elevato: le 
Geminiano Montanari, volendo dar conto degl' insabbiamenti e della direzione delle foei nella spiaggia veneta assume per fondamento teorico:

I. Che le maree accostano e discostano dal lido il moto radente senza interromperlo, oade nel riflusso le acque piegano a destra, ed ivi creano nei canali di acqua del mare la fossa subacquea :

II. Che rispetto ai flumi torbidi, questi lo trinciano a modo che rimane estinto sulla destra, e sulla sinistra si ac-

acque nel rif́asso non possono jnciderlo, o però piegano a destra, secondando la corrente dell' Adriatico, che il Monlanari osservò dírigersi da sivistra a destra.

- Quantunque autorevole sja per noi la testimonianza del Tadini, dubitiamo del falto recato che i porti di acqua dolce fendano per diritlo lo. scanuo, e si pieghino sulla destra quelli d' acqua salka. Vediamo i canali fátti dalla Marecchia, dalla Foglia; e dal Misa sulla costa adriatica fra Cesenatico o Senigallia avere la fossa mutabile secondo la direzione della traversia, come dicono, o del moto ondoso delle burrasche. Cosi gl' indicati canali che sboccano a tramontana, dopo le furiose burrasche di levante o greco-levante aprono la fossa in direzione composta fra tramontana o ponente, che rool díre a sinistra. Il porto di Cesenatico di sole acque salso. delle maree ha la fossa mutabile anch' esso; e dipendente dalla direzione della trapersia : ivi lo scanno è piu vicino alla foce ed elevato; ma per la stessa composizione dei movimenti del canale e delle burrasche, la fossa si trasporta qua e lá seconjo questa mutabile risultante.

- Vero per altro, che in tempo di quiete le acque del canale sortendo si gittano a sciroceo, o a destra, secondando la corrente adriatica del lido. Questa corrente osservata dal Montanari é poi si lenta, o quasi insensibile da non poter del certo escarare, e mantener sollevate, e trasportare le sabbie ancorchè tenuissime del fondo. Ognnno che abbia qual. che pratica delle coste adriatiche si sarà cogli occhi proprii assicurato di questo effetto, massime nelle bonacce estive, guardando alla visibil quiete di quel grande bacino d'acque. Secondo il Montanari, il moto radente sarebbe di M. 0.05 prossimamente per $1^{n}$, e questa sebbene piccolissima velocita, non abile a tener incorporate le torbide, ci sembra ancor soverchia, perchẻ ne' tempi della calma rediamo i galleggiantl più leggieri sensibilmente immobili, e quando si lera il moto ondoso il piu mite, seguire la direzione del rombo da cui muorono le onde. Peró dubitiamo di ogni teoria che pigli le cagionida quella corrente, e saremmo ritrosi ad ammettere le spiegazioni delle venete foci salse o dolci date dal Montanari, e dal Tadini, inchinando a pensare che la direzione del moto ondaso delle burrasche valga a guidarci con maggiore sicurezza nell' intendere questa maniera di fenomeni, e nell' applicarvi i rimedii ..

Vol. XIV: 
82

compagna, s' immedesima col corso del flume in mare; e là dove questo rimane estinto, il moto radente ripiglia il suo corso ricadendo sulla destra a maggiore o minore distanza secondo la portata del fiume; il quale deposita le sue torbide nello stagno creatosi, come sopra, da questa parte; ivi ingrossa il deposito a modo, che la fossa subacquea della foce $d$ obbligata a voltarsi, e piegare sulla sinistra.

Questa teoria fu accolta da sommi maestri, specialmente dallo Zendrini, e dal Manfredi nel proporre i miglioramenti pel porto di Ravenna, e da altri non meno insigni, come piü recentemente dal Venturoli parlando del porto d'Azio, e dal Paleocapa ne'ripetuti suoi scritti sulla corrente litorale.

Io confesso di non poter farmi capace del come il flusso e riflusso innalveato, che juò stimarsi a Malamocco di M. 1. 0 per 1", che fu trovato da me nel canale di Cesenatico di M: 0. 60, e nel Canal-bianco di M. 0. 47, non debba fare l'effetto di un flume torbido, a cui il Montanari concede velocità anche minore. Egli stesso si propone il dubbio: « Se nel riflusso sgor- gano le acque in mare, come quelle di un fiume, perchè non - fanno lo stesso effelto? Lo risolve dicendo, che le acque cor- rono fuori delle fauci dei fiumi verso il mare, come prove- nienti da parti superiori, e portate dal proprio natural im- peto che le spinge, onde tagliano la corrente del mare che - a loro si attraversa con più deboli forze, e ne riescono i - preaccennati effetti....

- Ma le acque delle lagune, e porti non discendono in ma* re se non quanto collo scemare nel riflusso, le acque marine - stesse tirano, per così dire, quelle che entrate erano col flus- so nelle lagune; che perd non è meraviglia, se queste cosi - attratte nell' uscire seguitano il corso del mare che le tira - da sinistra a destra..

A me pare che anche il riflusso incanalato corra appunto dall' alto al basso, come un fume, mosso similmente dalla pendenza superficiale del pelo d'acqua. Allo sbocco de' fiumi il fondo è naturalmente orizzontale, ed anche acclive, e tutto il moto dipende dalla inclinazione della superficie verso il mare, come ncl riflusso, il quale dovrebbe incidere, come il fiume; la correntia che gli si attraversa con piì deboli forze. 
Perchè poi un fume deve attirare, immedesimare a sè il moto litorale alla sinistra e non alla destra?

Chiunque osservi lo sboccare di un fiume in mare calmo, vede le sue acque seguitare col flone la direzione precedente, e la sua sezione traversa allargarsi in forma di ventaglio al di quà, e al di là di esso sempre piu, flno alla estinzione del suo moto; ivi formarsi il deposito delle torbide, o scanno che i mari burrascosi incidono, ora da una parte, ora dall' altra, aprendovi le foci subacquee variabili, e però di tanto pericolo alla navigazione.

Ricorda ognuno la celebre sperienza del Venturi, colla quale un getlo d'acqua attraversante l'acqua stagnante di un vaso. sottoposto le comunica il suo moto a destra e a sinistra tanto, ch'essa si accompagna col getto, e n'esce salendo, fuchè lascia vuoto il vaso sottoposto. Questa comunicazione laterale non sarebbe, secondo che io penso, alterata sensibilmente, ove si supponesse impresso un moto leggerissimo da destra a sinistra all' acqua del vaso sottoposto. E ciò penso, come cosa naturale e perchè a me pare che quella comunicazione laterale sia dovuta alla pressione atmosferica, che grava l'acqua stagnan. te, o lievemente mossa, ed è maggiore della pressione che ha l'acqua corrente del getto, nel sito ove si comunica con quella del vaso, sottoposto (1).

II Guglielmini (al cap. 8, cor. 7 propos. 4 sulla natura dei fumi ) nota * che gli sbocchi dei flumi nel mare sono ob- bligati a secondare le correntio di esso: siano queste perpetue, - o cagionate temporaneamente dai venti; quindi è che i fiumi - della Romagna, e del Ferrarese rivoltano gli sbocchi a de- stra, perchè la correntia dell'Adriatico rade il lido dell'Ita- lia, partendosi da Venezia verso la terra di Bari; ed in altri - luoghi i venti burrascosi obbligano i lumi ad aprirsi nuove - foci in luoghi coperti, o secondanti la furia di essi. Vero è - che le correntie del mare, se sono lente, non hanno gran - forza per cagionare l'effetto predetto; ma pure, quando nulla. - vi osti, non lasciano di fare quello che ponno .

(1) G. B. Venturi - Recherches experimentales sur la comunication laterale du mouvement dans les fuides ", Paris 1797. 
84

Ifo riportato per intero questo corollario, che cilai solamente nella mia Memoria, perchè si vegga, come questo discepolo insigne del Montanari (il quale conosceva le lagune venete, e il concetto del maestro) ne giudicasse la teoria. Anzi è da considerare il fatto recato e che i flumi del Ferrarese, e della Romagua voltano gli sbocchi a destra * al contrario di quello che dalla sua teoria ne deduce il Montanari, ed è poi generalmente più vero. Ma il Guglielmini guardando all' andamento della correntia, non poteva concluder altro, perchè quel tale stagno alla destra generato da una corrente lentissima che viene dalla sinistra alla destra, riesce impossibile da concepire, e contrario al fatto che può osservarsi facilmente da chicchessia. $\mathbf{E}$ non so che ad alcun altro sia caduto in mente quell'immedesimarsi da una parte sola dei fluidi urtanti cogli urtati negli sbocchi de' fiumi in mare, o nelle confluenze.

Quanto all' urto delle acque contro un ostacolo solido, stimava il Newton, che nell'urto diretto i fili fluidi perdessero successivamente tutto il moto, e ne deduceva la forza dell urto proporzionale al quadrato della velocità, e il Lagrange deduceva l'urto diretto di una vena contro una lastra, determinando la generatrice del conoide fluido che rimane stagnante simmetricamente intorno alla base, e all' asse della vena urtante; altri colla esperienza disegnava la prora fluida stagnante intorno all'asse dell'urto diretto, ed anche obliquo.

Quindi allorchè il flume si riguarda come un guardiano artificiale solido, secondo il parere del Montanari e di altri, e si conclude che gli effetti dell'uno, e dell'altro sono simili, mi pare che venga a dirsi che non può mancare anche dalla parte dell' urto uno stagno. Il fatto costantemente osservato è, che l' urto diretto contro un ostacolo solido piantato nella sponda cagiona una deviazione dei fili d' acqua dalla base alla punta, per cui ivi si genera un molente, o la prora stagnante che gli sperimentatori tutti avvertono. E chi ha veduti i pennelli normali al fllone che viene da sinistra a destra, più alti del pelo d'acqua, ha immancabilmente osservato l'interrimento che si fa dalla parte dell'urto, alla sinistra, più o meno vasto secondo la lunghezza dell' ostacolo. Si fa.l'interrimento anche alla destra, sc il pennello arriva a deviare totalmente il filone, ma se 
questo ricade rapidamente, l'interrimento alla destra è molto minore che alla sinistra; come può vedersi a Firenze, lungo l'arginestrada creato artificialmente nell'alveo dell'Arno per restringerne la sezione, e collocarvi il ponte di fli di ferro sospeso alle Grazie.

Quando il pennello è obbliquo al flone, allora l'interrimento diminuisce dalla parte urtata in proporzione della obbliquità maggiore o minore, verso la parte opposta, e si fa maggiore da questa parte.

Dalle considerazioni esposte risulterebbe, che la inflessione a destra della fossa subacquea del Canal-bianco non pud attribuirsi che alla Bora, da cui unicamente è dominata. Che prima del taglio di Porto-viro le foci del Po di tramontana e di levante fossero volte aila sinistra, era troppo consentaneo al fatto di tutte le altre foci dei fiumi inferiori. Que'due antichi rami del $P_{0}$ sboccavano allora in mare aperto, e non avevano la punta della maestra appoggiata al grandioso delta generatosi in seguito, che li riparasse dai venti del primo quadrante, onde obbedivano all'impeto delle levantare, sotto la qual denominazione i nostri marini intendono le burrasche fra greco e scirocco. Creatosi ora quel delta, come pud immaginarsi la corrente litorale non allontanata da quell' ostacolo sporgente sei o sette miglia dal lido aderente, ove sbocca il Canal-bianco, e dallo sbocco del Po dalla maestra, volendo seguire il concorde modo di vedere degli antichi maestri, e del Portolano?

Quel taglio o raddrizzamento fu fatto anche per allontanare le torbide del $P_{0}$ dalla foce dell'Adige, da Brondolo e da Chioggia, ov' erano trasporlate dalle traversie dominanti da greco a scirocco; cioè in senso contrario alla corrente litorale, che non bastava dunque ad allontanarle dalle venete foci! Ma non era allora sorta l'ipotesi del Montanari, che avrebbe consigliato a non pensare mai a quell' immenso lavoro.

Io sono sempre di credere, che dando al moto burrascoso del mare la potenza che ha di traslocare, e distendere lungo le coste i materiali portati dai fiumi, e sollevati dal fondo del mare, s'intendano immediatamente tutti quanti gli effetti, che succedono nelle spiaggie sottili cosi rispetto al protendimento del lido, come alla direzione delle fosse subacquee. $\mathrm{E}$ i fatti 
riferiti nella mia Memoria, mi sembrano più che sufficienti a far toccar con mano questa potenza del moto ondoso del mare, sia esso progressivo, continuo, o intermittente.

A que' fatti potrei aggiungere: che quando costruiamo un molo alle foci, lungo le spiagge Ferraresi, Romagnuole, Marchiane, vediamo subito insabbiarsi alla destra, al contrario di quello che accade nei lidi Veneti; se due, per armare compitamente lo sbocco di un canal chiaro, o torbido, il fatto permanente è che la protrazione sabbiosa del lido è sempre maggiore sul fanco del molo destro, che sul sinistro; e vi è notabile differenza, come ne'canali di riflusso del Bianco, e di Magnavacca in Comacchio, e di Ravenna, e di Cervia, e del Cesenatico. Ivi son chiare le acque, e quella corrente attratta, immedesimata col mare dovrebbe fare lo stesso effetto da ambe le parti, o più sulla sinistra adottando l'ipotesi del Montanari. Si avverta però che è alquanto più lungo il molo destro del sinistro, per facilitare l'ingresso alle barche, e ripararle alla bocca dalle traversie dominanti. Che importa cio in un moto di va-e-vieni parallelo ai moli, che porta e riporla le sabbic mosse dalle burrasche senza alterazione possibile dello stato precedente? Le sabbie mosse dalle levantare torneranno al loro posto; e se si dà alle levantare la facoltà che hanno di traslocarle, che importano altre considerazioni; non basta cio a capir subito che si fermeranno prima e in maggior copia alla destra che alla sinistra?

Ne'fiumi avendo mostrato che quello stagno a destra sembra troppo difficile da concepire, non conforme alle migliori teorie, e al fatto, che potrà farvi quella corrente lentissima? Aiutare tulto al più la via delle acque in mare se cospirante colla traversia prevalente, e se più o meno opposta allontanarsi finchè il moto del fiume resti estinto, per riprendere il naturale suo corso più al largo, e ricadere di nuovo sulla ripa.

In questa vicenda la fossa subacquea resterà sempre determinata dalla direzione della dominante forza del moto ondoso, e i deposili siano del fiume, o del mare si faranno a destra o a sinistra di quella foce, e in maggior copia da quella parte, d'onde provengono più abbondanti. La variabilità delle foci sian d'acque chiare o torbide, dolci o salate, deriva ap- 
punto dalla variabile direzione delle burrasche; e lo stato permanente è determinato, e proporzionale alla differenza degli effetti delle burrasche ordinarie sopra le straordinarie.

Le levantare sono prevalenti lungo la costa occidentale fra Ravenna ed Ancona, e provengono dalla destra alla sinistra, onde le foci son volte ordinariamente alla sinistra, e i maggiori protendimenti si fanno alla destra, le stesse traversie predominanti fanno l'effetto contrario nelle foci dalla punta di Sdobba al porto di Lido, pel contrario discorrimento della costa che investono da sinistra a.destra, riuscendo essa prossimamente perpendicolare alla prima (1).

(1) Sono in debito di pubblicamente dichiarare, che questo mio modo di vedere sulla corrente litorale lungo i lidi veneti, e la costa da Ravenna in Ancona, io inculcava al sig. Commendalor Cialdi dal 1842 al 1856. Egli mi mandò il suo manoscritto (che conserro ancora) sui cenni del moto ondoso del mare, ed io gli aggiunsi coraggio a pubblicarlo. Ivi riportó la mia opinione, e anche i fatti parziali da me osserrati che vi si leggono delle foci dell Adriatico. Onde se vi sia errore di concetto, o di osservazione, è tutto di mia colpa, e deve altribuirsi a troppa condiscendenza di lui verso di me. Il che bo voluto dichiarare per onor suo; o non arrei fiatato, se quel mio concetto non aresse incontrato autorevoli contraddizioni.

E poichè mi ha tratto l'occasione in questa materia, trascrivo qui letteralmente il rapporto che scrissi d' uficio sulla foce del Tevere, perchè mi parve anch' essa confermare il mio modo di redere, o presentare osservazioni di fatto non inutili a sapersi.

Romạa 26 Novembre 1842.

L'Ingegnere in Capo

Delle acque e strade di Roma.

A Sua Eminenza Reverendissima

Il sig. Card. Prefetto Generale delle acque e strade.

\section{Eminenza Reverendissima}

Vidi nello scorso Settembre, per l' Ingegnere in capo di Roma indisposto, i lavori di ordinario mantenimento del porto di Fiumicino, ed essendo it mare, e il fume nello stato ordinario, vi osservai una grande protrazione della spiaggia sinistra, che toccava la punta del guardiano da questa parte. La punta del guardiano opposto, M. 8. - piu sporgente in mare, era distante dalla spiaggia destra M. 51. -

Tutto il canale da Capo-due-rami topo alle indicate due estremita 
Aprendo il Portolano si vede immediatamente che da Sdobba a Lido la tramontana spinge al largo. Il greco prende tutto il Golfo fra Trieste e Venezia (il quale passato il promontorio

avea generalmente non meno di M. 3. d'acqua; oltr' esse in mare, attraverso il lido, di quà e di là sottile, una fossa per poca longhezza in dirittura dei guardiani, poi rivolta bruscamente a destra, e dopo breve tratto alquanto a sinistra; il cui fondo andava scemaudo mano mauo che si accostava allo scanno o prano, che sta rimpetto alla foce, e sorpassatolo non lasciava più traccia di se.

Questo giro tortuoso esterno si vedera, come suole, indicato dalle mee, per segno dell' unica via possibile a praticarsi dai naviganti che vanno e vengono.

Il minimo fondo $d^{*}$ acqua sulla sommilà dello scanno era in quel giorno N. 1. 30, appena sufficiente alle barche di 50, o 60 tonnellate.

Questo stato di cose rividi sul finire dell' Ottobre seguente, nell' occasione che col sig. Commend. Colonnello Cialdi visitai il Terere da Roma a Fiumicino.

Il peritissimo sig. Ingegnere Giorgi, che mi fu compagno l' una e I' altra volta, i pratici del luogo, e il lodato sig. Colonnello mi assicurarono, che tale era ordinariamente la condizione del detto canale $\theta$ della foce.

ond' è manifesto che deve riputarsi eccellente rispetto ai fondi d'acqua il canale stesso, ed infelicissima la foce; e che per la descritla direzione torluosa della fossa, questa difficilmente si trova in tempo di mare burrascoso, e di notte; nè mai senza pericolo di arenare, o di perdersi. Di che il lamento è generale, e fu cagione che V. E. R. mi ingiungesse di proporle il modo di provredervi.

Nell' adempiere ora a questa superiore di lei ingiunzione già tanto in sè importante, sentii di quanto maggiore momento era divenuta, dacchè introdotti i rimurchi a vapore, il commercio acquisterà notabile incremento per la speditezza dei cambi; e il concorso a Fiumicino si farà maggiore e con legni di più grosso carico.

Non vi ha fiume più atto alla navigazione interna di questo Tevere da Roma a Fiumicino; corre poco meno di due miglia e mezzo nostrane all'ora nello stato ordinario; con mitissima inclinazione di circa un palmo (M. 0.22) a miglio; ampio fra le ripe cento metri; col fondo costante di M. 1.50 nella massima magrezza ; con risvolte generalmente dolci e facilmente correggibili; di portata media il doppio della Senna a Parigi, un sesto del Pó; tragitla per l'alreo ogni anno la quarta parte della quantilà d'acqua che gli Elretici reggono passare pel Reno a Basilea; questo ingrossato dallo sfacimento di perpetue nevi, quello da due quinti delle tepide pioggio annuali della nostra temperata regione attraversa Roma! (")

(*) Estimatio aquae per alveum Tiberis praetergressae ab anno 1822 ad annum 1850. Josephi Venturoli, negli Alti dell' Istiluto di Bologna. 
di Salvore si allarga di molto), e cagiona un moto ondoso cospirante colla corrente litorale per lunga tratta, e con essa cospirano più o meno i venti intermedii fra greco e scirocco,

Da Roma verso le sue scaturiggini nella Falterona ḋ navigabile per 70 miglia fino a Ponte-Felice; e sebbene io non abbia ancor visto questo tronco, dalla magistrale relazione del Chiesa raccolgo, che mantiene condizloni non troppo diverse da quelle del ramo inferiore, onde i nuovi rimurchi posseno gia praticarlo, e lo praticano. Quanto no sarebbe lieta la S. M. di Benedetto XIV. che questa navigazione romana volera estendere fino a Perugia!

Ho toccate queste cose, perchè oltre accrescere peso anch' esse alI'ingiunzione di V. E. R. dimostrano, che lo stodio principale deve farsi alla foce, non sempre, né sicuramente praticabile.

Accade al Terere, ciò che a tutti i fumi del mondo, i quali sboccano in lido soltile; ingrossano la foce colle materie convogliate nelle piene, ed hanno innanzi, or più or meno lonlano, uno scanno o prano che fendono colle forze residue del corso precedente in direzione mutabile, secondo la direzione delle trarersie dominanti. La foce di Fiumicino guarda il poneate, e le ordinarie burrasche provengono dai venti meridionali; ond'è che la incisione dello scanno, e la fossa trovasi ordinariamente volta a destra, come assicurano i pratici ; e la brusca inclinazione cbe suole arere da questa parto proviene, per mio umile avriso, dai depositi del ramo d'Ostia spinti dalle trarersie dominanti addosso al guardiano sinistro attualmente interrato.

Lo stesso effetto abbiamo reduto langamente nel Lamone di Ravenna, che roltava la foce a destra, mentre tutti gli altri finmi della costa pontificia adriatica la voltano a sinistra ubbideudo all'ordinario dominio delle levantare; e questo arveniva perchè la punta del Po-Reno ivi prossimo a sinistra, si stende fino all' antica foce di quel fume, e l'obbligara a spandersi dalla parte opposta.

Per queste due cagioni degl' interrimenti del ramo d' Ostia, che giungono a Fiumicino, e dell' ordinario dominio dei venti meridionali, io credo che l'unico rimedio per migliorare la foce in discorso sia di prolungare it braccio sinistro, e di portarlo per ora almeno M. trenta più innanzi.

Mi confermano ancora in questa riverente opinione le osservazioni comunicatemi dall' Ingegnere del porto sig. Giorgi, dalle quali si può raccogliere, che la spiaggia sinistra cresce annualmente il doppio dell'allra, - la pratica osserrata costantemente nel nostri simiglianti canali dell' Adriatico, ne' quali il braccio destro è sempre più sporgente in acqua, appunto perchè da questa parte sono le burrasche di levante dominatrici di quel mare.

Questa protrazione per M. ̃̃0 almeno del guardiano sinistro accompagnerá il corso viro del canale ( come succede sempre lungo gli ostacoli 
che sono i dominanti. Quanto più investono direttamente $\mathrm{i}$ corsi d'acqua che sboccano in questa parte di costa, tanto più a mare medio tendono a versarsi sulla destra; e quando $i$ detti venti divengono opposti alla direzione di questi sbocchi tanto più ingolfano le acque a ritroso delle foci, e le ingrossano, come avriene per lo scirocco nei porti di Venezia, il quale fa crescere il pelo dell'estuario fino a sei piedi.

I ${ }_{0}$ scirocco avrebbe anzi una lieve tendenza d' inclinare le foci di Malamocco e di Chioggia a sinistra, ma le acque discendenti secondando la contraria direzione dei murazzi, e mantenendo la velocita preconcepita elidono, e superano quella lieve tendenza del pretto scirocco; il quale appena tende, come suole, al levante accresce lo sforzo da sinistra a destra, per cui si veggono quelle foci inclinate bruscamente da questa parte, e quasi parallele al lido per le acque del mare ingrossate che ne impediscono lo sgorgo libero, e la traversia che le piega da questa parte.

Ilo accennata la direzione generale della costa tra la punta di Sdobba e il porto di Lido, e fra Rarenna ed Ancona; ma nell'una, e nell'altra vi sono insenate più, o meno distese e specialmente fra Chioggia e Ravenna sulle quali, a intendere gl'insabbiamenti, e le direzioni delle fosse subacquee, convien ricorrere al dominio delle burrasche prevalenti, come nel Canal-bianco, guardando agli accidenti e alla esposizione dei rombi che le battono, piuttostochè alla corrente littorale, a non volere incontrare il falto in contraddizione colla teoria.

ll Montanari seguitando la sua ipotesi, consigliava a non allontanare la Piave da Cortelazzo e il Sile da S. Margherita

verticali) altraverso dello scanno, e la fossa si farà piú profonda e diritta, sarà meglio guardata dall" impeto delle ordinarie burrasche, impedirá lo spandimento delle materie del ramo d’ Ostia nella imboccatura, e farà che quelle di Fiumicino vadano a deporsi a destra nel bacino, largo $M$. 51 , che vi è da questa parte.

Siffatti beneficii non dureranno lungamente, o converrd mantenerli con periodiche protrazioni di ambo i guardiani, tenendo dietro agli annuali progressi delle due spiaggie. Né, per quanto io sappia, l'arte nostra può con altri divisamenti mantenere sufficientemente navigabili le foci de' fumi in lido sottile; e una lunga esperienza mi fa credere vana ogni opera diversa. (Segue lo scandaglio della spesa che si ommette). 
per arrestare $i$ sabbioni, che vengono da Sdobba in giù; al che son più potenti i flumi dei moli artefatti, perchè portano i sabbioni più lontano, e una parte se ne perde nei fondi alti; notava ancora che i flumi radunano più sabbioni alla destra', i reflussi alla sinistra. Ma sembra che le osservazioni di quel grande maestro rispetto alla Piave, e al Sile fossero troppo vicine alle diversioni operate di quelle acque, perchè guardando alle foci d'acqua salsa, o dolce, dalla punta di Sdobba in giù fino a Lido si trovano a cose stabilite segnate sulla destra ed $\mathbf{i}$ maggiori insabbiamenti sulla sinistra; veggansi le carte parziali del Portolano dal foglio I. al VI.

Quando si tratta della via d'acqua più facile, o della rotta dei bastimenti in mare, è indubitabile il fatto della tendenza e del moto lento delle acque marine dalla destra alla sinistra; onde si vantaggia entrando nel golfo a tenersi presso la costa orientale; e nel sortire presso la occidentale. Si guadagnano cosi tre o quattro miglia ogni 24 ore, avendo sempre cospirante il moto litorale, che corre appunto altrettanto. Supponiamo in ciò tranquillo il mare, e tanto meglio se mosso da vento cospirante. Ove questo fosse contrario, niun soccorso si avrebbe dal moto litorale, il quale, come nota il Portolano, muta direzione ad ogni lieve soffio di vento in direzione contraria; e l'ho verificato io stesso ne' piccoli galleggianti, pienamente sommersi, come riferiva nel citato mio articolo sul Tadini. Ció affermano costantemente anche $i$ nostri marinai, $i$ quali dicono che cogli sciroccali si rende insensibile il contrario effetto della corrente generale, e vanno in 12 o 14 ore a Venezia, e che basta una bava di questi venti a spedirsi di quella traversata.

Il Manfredi, e lo Zendrini volendo rendere ragione della perdita della foce navigabile del Candiano di Ravenna, messa da parte la corrente litorale, l'attribuivano, com'era naturale, al prossimo sbocco dei fiumi vecchi, rivolto contro di essa artificialmente, il quale protraendo il loro delta intorno alla foce, avea intasata quella del Candiano di sole acque di scolo chiaro; come il delta del Primaro non lontano ha fatto del Lamone costringendolo a piegare alla destra, contro la generale inclinazione di tutti i fiumi inferiori. Quanto alla foce della 
Bajona, nella carta unita alla loro relazione vedesi rivolta alla sinistra, contro la teoria del Montanari (1).

A me parve molto da considerare, e me ne espressi nella mia Nemoria, il falto del porto di Ancona, che imbocca la corrente litorale, e si mantiene da lanti secoli. Su di che il Paleocapa osserva, che la gittata recente del Lazzeretto difende ora il porto dall'ingresso delle torbide dei fiumi e della spiag. gia aderente; le quali, prima che fosse fatta, ne alzavano il fondo di un piede all'anno, come si nota nel Portolano. Non pare verosimile questo tanto alzamento in un porto che si mantiene da tanti secoli, e che fu ristaurato ed ampliato da Trajano, come si legge nell'Arco Romano erettogli sul molo vecchio; ed è si ben conservato fino a noi ! Le opere ordinarie di espurgo non sono del certo sufficienti a distruggere l'annuale alzamento di un piede veneto (M. 0. 35) sopra. M. q. 500000, che sono. oltre la metà di tutto il bacino; del quale interrimento anche soli M. 0.05 basterebbero in 100 anni ad ostruire affatto il porto.

Inoltre ançhe dopo la recente giltata, l'attual bocca del porto misura un'ampiezza di $M$. 460, onde con tanta apertura rimane alla corrente un'ampia e libera entrata per convogliarvi i materiali sollevati dalle burrasche.

La conservazione di quel porto mette in aperto, che essendo riparato dalle traversie dominanti, e anche dalla Bora, e aperto solo a ponente d'onde non procedon mai le burrasche, queste non vi recano che le torbide più lievi, e vi bastano gli espurghi ordinarii a mantenerne costanti i fondi: ivi il labro della imboccatura a sinistra è formato dalla punta di quella gittata, pesca in sette metri d'acqua, e dista M. 1500 circa dal Jido aderente.

Da queste circostanze del porto di Ancona a me nacque il pensicro di proporre un'opera simigliante artefatta per dare alla mia Rimino un porto a bacino, invece dell'attual portocanale alimentato dal torrente Marecchia; e mi par degno di essere discusso, se questa imitazione possa oltenere fiducia di

(1) Raccolta degli scrittori d' acque. Bolog a 1825, pag. 404, 405, 70lume 8. 
buon risultamento. Di che ho pregato voi, colleghi egregi, colla Memoria presentata al nostro Istituto nel luglio dell'anno passato, e contemporaneamente il Chiarissimo Commedator Paleocapa, ministro emerito dei lavori pubblici, e senatore del Reguo. Questi, a parer mio, diede una soluzione di fatto al mio quesito colla diga sinistra di Malamocco, e cooperando autorevolmeate, e con grande saper teorico, e pratico alla proposta dell'armatura della nuova foce di Saĩ $l$ nel canale di comunicazione da Suez al mediterraneo. Tanto la diga di Malamocco, che i proposti moli di Saïd si spingono in mare dal lido fino ai fondi alti, che occorrono alla grande navigazione. E gli effetti della diga di Malamocco hanno pienamente corrisposto all'aspettativa, nè crederei potersi dubitare di quelli che si aspettano dai moli di Saĩd.

Quando si arriva ai fondi alti oltre 8 metri, le agitazioni del mare non bastano a sconvolgere il fondo ancorchè sabbioso, e le sabbie sconvolte sul lido sottile non sono portate tanto al largo, onde o si accumulano alla riva, o si perdono per la massima parte negli alti fondi, o tutto al più si appoggiano agli ostacoli pì̀ o meno copiosamente in proporzione della loro quantità e dipendentemente dalla direzione dell' ostacolo, e delle burrasche che le trascinano. I moli si costruiscono ordinariamente normali al lido, $e$ il bisogno di allungarli è proporzionale al protendimento annuale del lido medesimo.

I moli come quelli di Malamocco, e del porto Saïd sono di alto costo, ma potendosi praticare in ogni spiaggia sottile naturalmente importuosa, ridurrebbero la questione di ottenervi un buon porto a bacino alla costruzione di moli più o meno lunghi in mare, e pero tutta la diffcoltà sarebbe della spesa.

La soluzione di questo quesito è di capitale importanza, perchè tutti $i$ paesi, grandi o piccoli situati in riva al mare vorrebbero un buon porto, e i Governi sono continuamente sollecitati da vivissime istanze per ottenerlo. Ma come a me pare che il moto ondoso del mare dia manifesta ragione delle ricorrenti difficolta che hanno gli approdi nelle nostre foci in lido sottile, siano chiare o torbide, salse o dolci, cosi stimo dispe. rato il caso di soddisfare a quelle istanze con opere di pocit spesa, come si è costrelti di fare dappertutto. Credo però, che 
ove il Governo, o le associazioni industriali sperassero di aprire una via a commerci importanti tolta la difficoltà della spesa, l'arte potesse sempre suggerire le opere necessarie a conseguire un successo sicuro. Invito i periti a pensarvi, perchè in questa eta, tutta rivolta agl'interessi materiali, l' argomento è di grande momento, e ignoro che sia stato trattato, come sembra di meritare.

Quanti studii, quante opinioni contrarie rispetto al porto d'Anzio, irreparabilmente perduto, e a tutti i nostri canali infelicissimi della costa occidentale!

La foce di Malamocco sarebbe oggi un pericolo anche maggiore di prima, se non riusciva al Paleocapa di raddrizzarla con quella diga grandiosa che va a trovare colla punta 12 o 14 metri d'acqua, e se non si desse opera di ampliarne il beneficio colla controdiga che si sta costruendo. Ivi il corso dei sabbioni dalle foci soltovento è copiosissimo: ma: quell'ostacolo. ne arresta la maggior parte sul lido sottile, e quella che $\dot{e}$ portata al largo va quasi tutta a perdersi negli alti fondi, ivi a mare quieto si deposita, e non può più risorgere sollevala dalle burrasche. Vi è di più il forte corso dei riflussi che basta a spazzare la piccola parte di quelle più sottili bellette, che rimangono più lungamente sospese nelle acque sconvolte, ed introdotle nel porto dalle burrasche; alle quali in mancanza di questo ajuto, come in Ancona, basta l'opera ordinaria de'cavafanghi. Cosi si mantiene coi fondi costanti questo porto; come io credo che si manterrà lungamente quello di Malamocco, al quale ad epoca lontana potrà tutto al più occorrere l'allungamento delle dighe. Dalla spiaggia più o meno torbida dipende, come accennava, la protrazione più 0 men sollecita dei moli, come la maggiore o minor lunghezza loro da principio. Crederei che queste difficolta dovessero essere minori dal Savio in Ancona, essendo quivi notabilmente minore l'annua protrazione del lido, che superiurmente. Infatti vediamo Adria, Ravenna, Rimini, citlà antichissime, questa lontana un miglio dal mare, le altre sette, e venti miglia, vi sono anche più prossime verso Ancona, Pesaro, e Senigallia.

Ma la materia è tanto vasta e grave, che da questi cenni non può essere chiarita, e io mi son lasciato andare a farli 
per invogliare chi si senta potente a trattarla con amore, parendomi che farà opera gloriosa, e di pubblico beneflcio.

\section{Conclusione.}

1. Raccogliendo le suesposte considerazioni, risulterebbe che la teoria del Montanari rispetto alla corrente litorale insumiciente, e non occotre a dare spiegazione agl'insabbiamenti delle foci, e alla direzione delle fosse subacquee, $e$ in generale all' annuo protendimento delle nostre spiagge sottili.

Vediamo le traversie dominanti abili a trasportare e a distendere lungo la costa le ghiaje vomitate alle foci dai torrenti, come può rimaner dubbio che non sian abili a distendere nel senso loro anche le grosse sabbie, le più sottili, e le finissime torbide? e a far piegare gli sbocchi dei canali, e dei flumi? Onde senza ricorrore alla lenta correntia generale s'intedono subito gl'insabbiameuti, e le fosse subacquee della costa occidentale, di qualunque natura siano.

2. Che anzi la corrente litorale ahile solamente a traslocare le torbide finissimo, che rimangono più lungamente sospese nelle acque del mare sconvolte, non lascia intendere, come la sua opera quantunque continua, e sempre nello stesso senso, possa bastare a produrre effetti contrarii e permanenti, come si osservano nei nostri sbocchi armati o disarmati che siano. Cessata la burrasca, dopo qualche tempo anche il limo fnissimo cade al fondo; nè può rialzarlo quella troppo lenta corrente continua, vi occorre una nuova burrasca che lo sollevi di nuovo, e lo rimetta in azione; ma se la nuova burrasca ha direzione contraria, non solo distrugge il suo fatto precedente, na ne produce uoo contrario, traslocando dalla parte opposta il limo depositato prima, e non di rado anche una parte del letto su cui posava.

A riprodurre quel primo fatto vi vorrebbe, oltre l'opera costante della corrente, quella eziandio di una burrascia cospirante con essa; e ciò costantemente, il che è contro a yuanto accade. Onde il risultamento finale sarà sempre derivato dalla prevalente direzione del moto ondoso del mare sia progressivo continuo, o intermittente. Se questo come notavamo, cospirera 
96

colla correntia crescerà di potenza, se sari opposto eliderà quello della correntia, e produrrà effetti opposti.

É poi si tenue l'aiuto che può dare al moto burrascoso l'azione di quella corrente anche cospirante, che non bisogna il suo concorso a intendere gli effetti che si veggrono, onde a me parve di poter concludere, che senz' essa i protendimenti del nostro lido, e la disposizione delle foci sarebbero, come sono.

Vero è che una hurrasca ancora può distruggere l'effetto della precedente se procede da opposta direzione, ma le levantare nella parte di costa che esaminiamo, essendo predominanti e ricorrenti ogni anno, superano gli effetti delle burrasche contrarie che debbono riguardarsi straordinarie specialmente riguardo agli effetti di cui si tratta. F quando, a cagion d' esempio, per qualche parziale accidente della spiaggia, una foce sia esposta all'impeto dei venti boreali, e riparata dagli altri, dia quelli dipenderanno gl'insabbiamenti, e la direzione della fossic subacquea, come nel Po di levante, o Canal-bianco.

$3 .^{\circ}$ Crederei finalmente che colla stessa guida delle traversie prevalenti potesse intendersi facilmente, che il punto di massimo insenamento del lido fra due foci torbide si stabilisse ove giungono le minime quantità di limo dei due fiuni, onde al porto di Volano toccano gl'insabbiamenti dal Po e non dal. Primaro-Reno, a quello di Magnavacca dal Primaro-Reno e non dal Po; cosi al porto Corsini più dai flumi uniti che dal Primaro-Reno, dacchè il Lamone fu gittato belle valli; e similmente al canale di Cesenatico ugualmente dagli sbocchi inferiori che dai superiori.

So che questo mio modo di vedere in questi fenomeni si discosta forse troppo da quello di tanti insigni maestri, ma sento nell'animo che procede unicamente dal mio vivo desiderio d'intenderli, onde spero, che ove non possa essere accolto, sarà almeno scusato. 